\title{
Reorganização do serviço ambulatorial de referência para condiçōes crônicas durante a pandemia da COVID-19
}

\author{
Reorganization of the outpatient reference service for chronic conditions during the COVID-19 pandemic \\ Reorganización del servicio de referencia ambulatoria para condiciones crónicas durante la pandemia \\ de COVID-19
}

Francielle Renata Danielli Martins Marques ${ }^{1}$ (1) Lilian Ferreira Domingues ${ }^{1}$ (C)

Lígia Carreira $^{1}$ (1) Maria Aparecida Salci ${ }^{1}$ (D)

1. Universidade Estadual de Maringá. Maringá, PR, Brasil.
Autor correspondente:

Francielle Renata Danielli Martins Marques. E-mail: franrenata.martins@gmail.com

Recebido em 13/09/2021.

Aprovado em 30/11/2021.

\section{Resumo}

Objetivo: Relatar a experiência da equipe de saúde da atenção especializada na reorganização do processo de trabalho para a continuidade do cuidado às pessoas com condições crônicas complexas durante a pandemia da covid-19. Métodos: Relato de experiência vivenciada em ambulatório do Estado do Paraná entre março e julho de 2020. O serviço ambulatorial em questão adota o Modelo de Atenção às Condições Crônicas para o atendimento de gestantes, crianças, idosos, pessoas com hipertensão arterial, diabetes mellitus e transtornos mentais, estratificadas como condições complexas. Resultados: $\mathrm{O}$ avanço da pandemia no Brasil implicou a necessidade de planejar a reorganização da atenção ambulatorial especializada, definindo atividades assistenciais presenciais no serviço, atividades itinerantes nos municípios e uso de tecnologias remotas para assistência e matriciamento. Conclusão e Implicações para a prática: $O$ rigor no cumprimento das recomendações sanitárias possibilitou a reorganização dos processos de trabalho no serviço, com modificações que permitiram a continuidade do cuidado de pessoas com condições crônicas complexas. O planejamento e o desenvolvimento das modificações no serviço foram fundamentais para manter o acompanhamento e o monitoramento da saúde das pessoas com condições crônicas complexas em meio a pandemia, minimizando as descompensações e, consequentemente, diminuindo a necessidade de essas pessoas utilizarem os serviços de saúde.

Palavras-chave: Assistência Ambulatorial; Covid-19; Doença Crônica; Enfermagem; Infecções por Coronavírus.

\section{Abstract}

Objective: To report the experience of the specialized care health team in reorganizing the work process for the continuity of care for people with complex chronic conditions during the COVID-19 pandemic. Methods: Experience report lived in an outpatient clinic in Paraná State between March and July 2020. The outpatient service in question adopts the Chronic Conditions Care Model for pregnant women, children, elderly people, people with hypertension, diabetes mellitus, and mental disorders, stratified as complex conditions. Results: The advance of the pandemic in Brazil implied the need to plan the reorganization of specialized ambulatory care, defining face-to-face care activities in the service, itinerant activities in municipalities, and the use of remote care technologies and matrix support. Conclusion and Implications for practice: Rigorous compliance with health recommendations allowed the reorganization of work processes in the service with modifications that allowed continuity of care for people with complex chronic conditions. The planning and development of the modifications in the service were fundamental to maintain the follow-up and monitoring of the health of people with complex chronic conditions amid the pandemic, minimizing decompensations and, consequently, reducing the need for these people to use health services.

Keywords: Outpatient Care; COVID-19; Chronic Disease; Nursing; Coronavirus Infections.

\section{Resumen}

Objetivo: Exponer la experiencia del equipo de atención a la salud especializada, en la reorganización del proceso de trabajo para la continuidad de la atención a personas con enfermedades crónicas complejas durante la pandemia de COVID-19. Métodos: Informe de experiencia en un centro ambulatorio del estado brasileño de Paraná entre marzo y julio de 2020. El servicio adopta el Modelo de Atención a Condiciones Crónicas para el atendimiento de: gestantes, niños, ancianos, personas con hipertensión arterial, diabetes mellitus y trastornos mentales, estratificado como condiciones complejas. Resultados: El avance de la pandemia en Brasil implicó en la necesidad de planificar la reorganización de la atención ambulatoria especializada, al definir acciones de actividades asistenciales presenciales en el servicio, actividades itinerantes en los municipios y el uso de tecnologías remotas para la asistencia y apoyo matricial. Conclusión e implicaciones para la práctica: El rigor en el cumplimiento de las recomendaciones sanitarias permitió la reorganización de los procesos de trabajo en el servicio, con cambios que permitieron la continuidad del cuidado de las personas con condiciones crónicas complejas. La planificación y el desarrollo de los cambios en el servicio fueron fundamentales para mantener el acompañamiento y la vigilancia de la salud de personas con condiciones crónicas complejas en medio a la pandemia, minimizando las descompensaciones y, consecuentemente, disminuyendo la necesidad de que estas personas utilicen los servicios de salud.

Palabras clave: Atención Ambulatoria; COVID-19; Enfermedad Crónica; Enfermería; Infecciones por Coronavirus. 


\section{INTRODUÇÃO}

A covid-19 é uma doença causada pelo coronavírus SARSCoV-2 (Severe Acute Respiratory Syndrome Coronavirus 2), que traz inúmeras ameaças à saúde pública mundial. A doença adquiriu o status de pandemia em 11 de março de 2020 e tem sido considerada como um dos maiores desafios sanitários deste século. ${ }^{1}$ As infecções por coronavírus são decorrentes da transmissão do vírus por gotículas respiratórias ou contato, sendo necessários cuidados especiais referentes à etiqueta respiratória, lavagem de mãos com água e sabão, além da redução da circulação de pessoas em vias públicas para evitar a disseminação da doença. ${ }^{2}$

O Brasil é o terceiro país com mais casos de covid-19 no mundo, sendo o segundo em óbitos: até o final da $25^{a}$ semana epidemiológica de 2021, havia 18.386.894 pessoas infectadas e 512.735 óbitos no país. Nessa estatística, destaca-se o estado do Paraná, que apresentou a segunda maior taxa de óbitos no Brasil, com 13,7 óbitos/100 mil habitantes. ${ }^{3}$

Diante dessa situação pandêmica, os serviços de saúde precisaram se reorganizar para atender às pessoas com doença crônica e em condições de saúde que demandam acompanhamento contínuo. Profissionais do Serviço de Atendimento Móvel de Urgência (SAMU) de uma capital da região Sul do Brasil revelaram mudanças nos fluxos de atendimento e nas rotinas laborais, bem como novas necessidades de precaução biológica e suporte emocional para os profissionais envolvidos. ${ }^{4}$ No contexto das condições crônicas, os profissionais de saúde têm um papel fundamental em manter a motivação das pessoas na adesão ao tratamento medicamentoso e a mudanças de comportamento, como dieta e exercícios físicos. ${ }^{5}$

Nesse contexto sanitário, a Atenção Ambulatorial Especializada (AAE), que integra o nível secundário do Sistema Único de Saúde (SUS) e sistematiza sua atenção às especificidades do Modelo de Atenção às Condições Crônicas (MACC), ${ }^{6}$ precisou se readequar para manter $\mathrm{o}$ acompanhamento das pessoas com condições crônicas complexas durante a pandemia. O MACC se tornou referência pelo Ministério da Saúde para o desenvolvimento das Redes de Atenção à Saúde (RAS) que realizam atendimento às pessoas em situação de cronicidade. ${ }^{6}$

Conforme o MACC, as competências da AAE consistem em oferecer retaguarda assistencial e apoio educacional para a equipe da Atenção Primária à Saúde (APS). ${ }^{6}$ As ações assistenciais são oferecidas para as pessoas estratificadas com condições crônicas complexas, e o apoio matricial ocorre por meio de consultorias técnico-pedagógicas. É imprescindível o conhecimento mútuo de ambas as equipes para que diferentes saberes sejam somados no trabalho conjunto.

Como as pessoas com condições crônicas são consideradas grupo de risco para a covid-19, ${ }^{1}$ a AAE precisou ajustar sua agenda, rotinas, protocolos e espaços físicos para prestar um atendimento seguro a esse público. Também foram utilizadas ferramentas da telemedicina, como chamadas de vídeo e ligações telefônicas. ${ }^{7}$
Diante desse contexto, este relato buscou descrever as modificações que a equipe de saúde especializada realizou no seu processo de trabalho para continuar prestando assistência às pessoas com condições crônicas complexas e manter o suporte educacional por meio do apoio matricial às equipes de saúde da APS, conforme o MACC. Assim, este estudo objetivou relatar a experiência da equipe de saúde da atenção especializada na reorganização do processo de trabalho para a continuidade do cuidado às pessoas com condições crônicas complexas durante a pandemia da covid-19.

\section{MÉTODOS}

Estudo do tipo relato de experiência sobre a atuação de uma equipe de saúde de AAE na reorganização dos processos de trabalho durante a pandemia da covid-19. O relato de experiência é concebido como um estudo de natureza qualitativa, que valoriza a explicitação descritiva, interpretativa e compreensiva de fenômenos, delegando, ao pesquisador, o desafio de articular conhecimentos teóricos com as interpretações do fenômeno relatado. ${ }^{8}$

Para a compreensão dos fenômenos, adotou-se a técnica de observação com participação moderada, permitindo que o pesquisador se inserisse no contexto observado e interagisse com os participantes, o que viabilizou apreender o significado de estar naquela situação. ${ }^{9} \mathrm{~A}$ observação moderada ocorreu nos atendimentos individuais e nas rotinas administrativas de todos os profissionais da AAE, a fim de que fossem identificadas as mudanças ocorridas na estrutura organizacional da AAE e no funcionamento dos serviços de atendimento ofertados às pessoas com condições crônicas durante a covid-19. Para além da observação, foram agendados cinco momentos de discussões com a direção e a enfermagem para a atualização das informações sobre a reorganização do serviço. Esses momentos ocorrerem por chamada de telefone.

Os processos descritos neste estudo ocorreram no período de março a julho de 2020, na AAE localizada em um município da região centro-norte do Estado do Paraná. A AAE em questão é referência para atendimentos especializados do Sistema Único de Saúde (SUS) para os 17 municípios que pertencem à $16^{\mathrm{a}}$ Regional de Saúde, o que corresponde a uma população de quase 393.000 pessoas. ${ }^{10} \mathrm{O}$ serviço implementa o MACC desde 2016 para atender pessoas em situação de cronicidade, estratificadas pela APS conforme as RAS prioritárias: Gestantes, Crianças, Idoso, Hipertensão Arterial, Diabetes mellitus e Saúde Mental. Para $o$ atendimento, o serviço conta com equipe multiprofissional composta por 23 pessoas das seguintes categoriais profissionais: Assistente Social; Enfermeiro; Fisioterapeuta; Fonoaudiólogo; Médicos com especialidades em cardiologia, endocrinologia, geriatria, obstetrícia, pediatria e psiquiatria; Nutricionista; Psicólogo; e Pedagogo.

No período pré-pandêmico, o atendimento das RAS era realizado de forma presencial e individual, de segunda à sexta-feira, nos turnos de manhã e tarde. Por meio da atenção contínua, a equipe multiprofissional se organizava para prestar 
atendimento sequencial às pessoas com condições crônicas complexas que compareciam à AAE. A RAS do Idoso era a única que não realizava atendimento na sede da $A A E$, organizando-se de modo que a equipe multiprofissional se deslocava, de forma itinerante, até os municípios para realizarem o atendimento das pessoas in locu.

Além dos atendimentos assistenciais, o serviço realiza ações de apoio matricial, como suporte para a gestão do cuidado $e$ educação permanente aos profissionais da APS. Assim, os processos de trabalho que precisaram ser ajustados durante a pandemia envolveram aspectos assistenciais e matriciais, tendo sido desenvolvidos de forma presencial, itinerante ou remota.

Este relato é resultado da inserção de uma pós-graduanda no serviço de AAE. Nos meses que precederam a pandemia, a inserção ocorreu de forma presencial; com o avanço da covid-19, o acompanhamento foi remoto. Esse é o cenário da pesquisa de dissertação de Mestrado aprovada pela Comissão Permanente de Avaliação de Projetos e do Comitê Permanente de Ética em Pesquisa com Seres Humanos (COPEP), conforme Parecer $n^{\circ}$ 4.032.609, CAAE: 27998719.8 .0000 .0104 , de acordo com as Resoluções 466/2012 e 510/2016 do Conselho Nacional de Saúde sobre pesquisas com seres humanos.

\section{RESULTADOS}

A fim de melhor apresentar a reorganização do processo de trabalho na AAE com a pandemia da covid-19, os resultados deste estudo foram agrupados em três categorias: 1) planejamento para a reorganização da $\mathrm{AAE} ; 2$ ) atividades assistenciais presenciais na $A A E$ e atividades assistenciais itinerantes nos municípios; $\mathrm{e} 3$ ) utilização de tecnologias remotas para assistência e matriciamento.

\section{Planejamento para reorganização da atenção especializada}

Em 23 de março de 2020, os atendimentos presenciais na AAE foram suspensos por duas semanas, com a recomendação de que a equipe permanecesse nos seus respectivos domicílios. A partir de 06 de abril de 2020, a equipe multiprofissional retornou à AAE para planejar, juntamente com a direção, alternativas para minimizar o impacto da suspensão/diminuição dos atendimentos às pessoas com condições crônicas que estavam em acompanhamento periódico no serviço.

Ficaram definidas as seguintes estratégias assistenciais: atendimentos presenciais na $\mathrm{AAE}$, atendimentos presenciais itinerantes nos municípios e utilização de tecnologias remotas para assistência e apoio matricial às equipes da APS. Diante da necessidade de atender a população idosa, a qual se configura como a mais vulnerável à covid-19, os atendimentos que a equipe da $A A E$ realizava de forma itinerante nos municípios foram ampliados para todas as RAS. Além disso, alguns transportes municipais de saúde não puderam conduzir pessoas acima dos 60 anos de idade, gestantes e crianças, o que, para as RAS, afetou diretamente a quantidade de pessoas atendidas no serviço.
Ademais, a equipe se reorganizou para manter o apoio matricial para a APS de forma remota, por meio de ligações telefônicas, chamadas de vídeo, mensagens de texto e e-mails.

\section{Atividades assistenciais presenciais na atenção especializada}

A retomada dos atendimentos presenciais implicou reorganização estrutural para receber as pessoas de forma segura. O serviço distribuiu almotolias com álcool $70 \%$ em pontos estratégicos, demarcou o distanciamento de $1,5 \mathrm{~m}$ entre as pessoas e proibiu o uso do ar condicionado, priorizando manter as janelas abertas para favorecer o fluxo de ar.

Foram designados técnicos de enfermagem, devidamente capacitados e paramentados, para realizarem a triagem individual de todas as pessoas na porta de entrada que antecede o acesso às instalações do serviço. Nesse momento, os profissionais realizavam a anamnese direcionada aos sintomas gripais e/ ou contato com pessoas gripadas e verificavam a temperatura.

Os casos suspeitos de covid-19 eram redirecionados à APS de origem, atendendo às recomendações sanitárias. O reconhecimento dos casos suspeitos antes mesmo de as pessoas adentrarem o serviço se constituiu numa rotina primordial para evitar a disseminação do vírus em sala de espera, assim como para proteger as demais pessoas em atendimentos e a equipe de saúde. Contudo, ressalta-se que não houve identificação de casos suspeitos na AAE, o que reforça a qualificação da triagem prévia realizada pela APS, uma vez que só era realizado agendamento para pessoas sem suspeitas da covid-19.

$\mathrm{O}$ agendamento das consultas presenciais foi realizado com horários espaçados para evitar aglomerações nas salas de espera. Esses agendamentos eram solicitados pela APS para os casos em que se reconhecia agravamento no quadro clínico da pessoa com condição crônica. Uma vez solicitado o agendamento pela APS, a equipe multiprofissional da AAE era responsável por fazer a regulação das agendas e confirmar o agendamento presencial, encaminhando as informações para a APS, como data e hora agendadas. Somados, o número de pessoas atendidas presencialmente pelas RAS foi reduzido em $60 \%$, passando de aproximadamente 300 para 180 pessoas atendidas por semana.

O uso de máscara de pano ou cirúrgica foi exigido para todas as pessoas. Já para a equipe multiprofissional, foi exigido o uso de máscara cirúrgica, luvas, óculos ou face shield e avental.

Cada atendimento gerou como produto um plano de cuidados multiprofissionais, conforme proposto pelo MACC. Havia pactuações de saúde, metas clínicas e prescrições medicamentosas que continuaram a ser compartilhadas com a equipe da APS de forma remota, como era realizado no período pré-pandêmico.

\section{Atividades assistenciais itinerantes nos municípios}

Os atendimentos itinerantes nos municípios passaram a ser realizados por todas as RAS e ocorriam na sede da Unidade Básica de Saúde (UBS). Previamente, a enfermeira da UBS 
entrava em contato com as enfermeiras responsáveis por cada RAS na AAE, enviando as estratificações de risco das pessoas com condições crônicas instáveis. Cada documento era analisado, era conferida a estratificação e programada a consulta. Essa programação ocorria em acordo com a APS, a qual também era responsável por avisar as pessoas que seriam atendidas e planejar um local adequado para esses atendimentos.

$\mathrm{Na}$ data programada, a equipe multiprofissional da AAE se deslocava até a UBS, que já estava organizada para recebê-la. Cada profissional ficava em um consultório para que pudessem realizar a atenção contínua, com atendimento individualizado e sequenciado, assim como ocorria na AAE.

Os profissionais da UBS também compareciam para acompanhar os atendimentos da equipe especializada, no formato de atendimento compartilhado vinculado às ações de apoio matricial. Nessa reorganização, foi possível que tanto as atividades assistenciais como o suporte educacional fossem realizadas concomitantemente.

Antes das consultas, realizava-se a avaliação de pessoas para triagem de problemas respiratórios, a qual era feita por profissionais da enfermagem da UBS. As pessoas com atendimento agendado precisavam utilizar máscara de pano ou cirúrgica, e a equipe multiprofissional utilizava máscara cirúrgica, luvas, óculos ou face shield e avental. Após a finalização dos atendimentos, 0 produto era um plano de cuidados, elaborado conforme o MACC e já entregue à pessoa, além de ser compartilhado com a APS.

No período compreendido pelo estudo, foram realizadas 22 atividades itinerantes em UBS localizadas em 11 municípios, das quais participou toda a equipe multiprofissional, cujos profissionais se organizaram em sistemas de rodízio.

\section{Uso de tecnologias remotas para assistência e matriciamento}

Pelo fato de fazerem parte de grupos de risco, muitas pessoas não puderam retornar às consultas presenciais no início da pandemia. Sendo assim, as RAS de Hipertensão Arterial, Diabetes mellitus, Gestantes, Crianças e Idosos foram amplamente atingidas, já que, pelas próprias condições de saúde, foram consideradas de risco para possíveis complicações da covid-19. Por isso, alternativas de atendimentos e monitoramentos não presenciais, mediados pelo uso de tecnologias de comunicação virtual ou remota, foram implementadas no serviço, sendo que as ligações telefônicas foram os recursos mais utilizados pelo serviço.

Para a realização das atividades remotas, a equipe multiprofissional se redistribuiu, organizando-se em grupos que faziam consultas remotas às pessoas com condições crônicas e em grupos que realizavam contato com as equipes da APS para apoio matricial. Não houve divisão fixa para a execução dessas tarefas, tendo sido realizado rodízio entre todos os profissionais, com uma programação de agenda prévia.

Em relação às consultas remotas, os profissionais faziam questionamentos sobre as práticas de autocuidado, como uso das medicações contínuas, alimentação, atividades físicas no domicílio, além de orientações sobre o reconhecimento dos sinais e sintomas precoces da covid-19. As pessoas também foram questionadas sobre saúde mental durante a pandemia.

Todos os atendimentos profissionais tiveram que ser readequados ao cenário pandêmico. Os profissionais de saúde apresentaram opções para exercícios no domicílio, principalmente no caso dos idosos frágeis, em que a mobilidade precisava ser incentivada diariamente. Entretanto, uma das principais queixas das pessoas era em relação ao sofrimento mental ocasionado pelas restrições e medidas de isolamento social recomendadas pela OMS para a segurança sanitária durante a pandemia da covid-19. Assim, diante do relato de sofrimento, era oferecido maior suporte emocional, com escuta qualificada, permitindo que a pessoa verbalizasse suas angústias e sofrimentos. As pessoas com sinais e sintomas que indicassem maior fragilidade eram direcionadas à psicóloga do serviço.

Em relação ao suporte para a APS, foi mantido o apoio técnico-pedagógico do matriciamento, realizado de acordo com - MACC; porém, esse apoio era dado a distância. O suporte para a APS funcionava como uma "segunda opinião"6 de especialistas para os casos de maior dificuldade de manejo da APS. Além disso, os momentos de conversas entre as equipes da AAE e APS serviam para discutir casos clínicos, adesão ao plano de cuidados, reformulação dos planos para atender às necessidades das pessoas, monitoramento das pessoas com condições crônicas estabilizadas e com condições instáveis. Tudo era feito a fim de traçar novas metas ou agendar atendimento às pacientes de difícil manejo.

A fim de manter a realização das atividades remotas, todos os atendimentos eram registrados no prontuário clínico do paciente, e as atividades de suporte para a APS eram registradas em documento próprio, já utilizado pela equipe. $\mathrm{O}$ número aproximado de atendimentos remotos realizados pelas RAS chegou a, aproximadamente, 150 pessoas por semana.

\section{DISCUSSÃO}

De acordo com o MACC, ${ }^{6}$ as pessoas com condições crônicas recebem uma atenção fundamentada em três pilares: 1) estratificação de risco, realizada conforme as linhas guia, cujo estrato de baixo, moderado e alto risco garantem o acesso ao serviço necessário; 2) autocuidado apoiado por profissionais de saúde, com ações direcionadas ao protagonismo das pessoas para aprenderem a cuidar de si; e 3) plano de cuidados, com metas de saúde para que a pessoa alcance a estabilização das suas condições; tais planos são elaborados por equipes multiprofissionais e acordados com as pessoas atendidas.

O avanço da pandemia da covid-19 no Brasil repercutiu nos serviços de saúde, de modo que foi necessário ele se reorganizassem a fim de preservar a saúde das pessoas atendidas. Ainda, para que as pessoas com condição crônica não ficassem desamparadas pelos profissionais, uma vez que essas condições, mesmo que estabilizadas, demandam cuidado e monitoramento contínuos. 
Sendo a $A A E$ responsável pela assistência às pessoas com condições crônicas complexas, ${ }^{6}$ foi necessário reestruturar rapidamente seus serviços como uma forma de ofertar a continuidade do cuidado às pessoas assistidas e, consequentemente, evitar a sobrecarga ao setor terciário, já tão atingido pela pandemia.

Assim, foi estabelecido que a AAE mantivesse suas atividades, mas que reorganizasse seus processos de trabalho conforme o grau de estabilidade da população, o qual variou das condições crônicas estabilizadas até a instabilidade persistente com presença de Síndrome Gripal ou Síndrome Respiratória Aguda Grave. ${ }^{11}$ Uma vez identificados os graus de estabilidade, as pessoas com condições crônicas instáveis receberam atendimento individualizado pelos profissionais da AAE, fosse remoto ou presencial; já as pessoas com condições estabilizadas mantiveram o acompanhamento na APS sob a supervisão da AAE. ${ }^{11}$

Para as pessoas com condições instáveis, os atendimentos realizados de forma presencial no serviço especializado seguiram protocolos vigentes que, além das precauções padrão, solicitavam implementar, adicionalmente, as precauções para contato, precauções para gotículas e precauções para aerossóis. ${ }^{12}$

Os resultados apresentados evidenciaram que o serviço manteve as ações para a continuidade do cuidado, com acompanhamento longitudinal das pessoas com condições crônicas complexas, de acordo com seu estrato de risco, estabilidade clínica, capacidade de autocuidado e suporte profissional para o enfrentamento da pandemia. Tal suporte ocorreu, principalmente, por meio do monitoramento do plano de cuidados a distância, com o auxílio da APS, conforme as orientações normativas.

Neste momento pandêmico, sobressai-se a importância do trabalho da equipe multiprofissional de saúde, a qual reorganizou suas práticas assistenciais para proporcionar desfechos satisfatórios às pessoas atendidas. ${ }^{7}$ Como destaque dessa reorganização, predominaram as consultas remotas realizadas por ligações telefônicas. Pessoas com mais idade e com nível educacional mais baixo apresentavam dificuldades em utilizar outras ferramentas para atendimento remoto. ${ }^{13}$

Os atendimentos, mesmo que na modalidade remota, foram meios de motivar a adesão à terapia medicamentosa e a mudanças de comportamento, necessárias para tratar o quadro crônico. ${ }^{5}$

Além da assistência, o serviço manteve as atividades de apoio matricial para orientação dos casos que permaneceram sob monitoramento da APS. O trabalho remoto foi organizado para apoiar a APS como suporte técnico-pedagógico na orientação dos casos que permaneceriam sob monitoramento da APS. ${ }^{14}$ Discussão conjunta de casos, construção do plano de cuidados $r$ "segunda opinião" dos especialistas foram ações realizadas por meio de ferramentas remotas, ${ }^{11}$ contribuindo para a manutenção da continuidade do cuidado, conforme o MACC.

Considerando que a finalidade primordial durante esta pandemia é priorizar a redução de riscos à saúde, além de proporcionar desfechos satisfatórios para as pessoas com condições crônicas complexas, relatos como este são necessários para fornecer informações, compartilhar estratégias e descrever reorganizações dos processos de trabalho durante o período pandêmico, o que pode auxiliar outros serviços de saúde.

\section{CONCLUSÕES E IMPLICAÇÕES PARA A PRÁTICA}

O relato apresentou a experiência da equipe de saúde da atenção especializada na reorganização do processo de trabalho, a fim de dar continuidade ao cuidado às pessoas com condições crônicas complexas durante a pandemia da covid-19. Os resultados demonstraram que nenhuma pessoa com suspeita da covid-19 foi agendada para a $A A E$, e os agendamentos respeitaram o espaçamento de horários, tendo sido implementadas medidas de proteção para a equipe e as pessoas atendidas, demonstrando rigor no cumprimento das recomendações sanitárias.

A integração da equipe multiprofissional para o desenvolvimento do trabalho foi fundamental, uma vez que ela participou das decisões junto à direção do serviço. Além disso, teve autonomia para se organizar e reestruturar os atendimentos presenciais na AAE (itinerantes nos municípios ou remotos), com destaque para a manutenção das atividades de apoio matricial para a APS. Este relato demonstrou, portanto, que as modificações ocorridas no processo de trabalho da AAE durante a pandemia permitiram a continuidade do cuidado das pessoas com condições crônicas complexas.

Dos limites do estudo, destaca-se a carência de pesquisas sobre a reorganização da assistência ambulatorial que atenda pessoas em situação de cronicidade, o que impossibilitou uma discussão mais ampla. Também é pertinente apontar as limitações impostas pela própria pandemia, o que repercutiu na suspensão da permanência da pesquisadora no serviço, sendo necessário recorrer aos aplicativos para videoconferência ou ligações por chamada de vídeo para que a pesquisa fosse finalizada sem perder a qualidade do produto final.

Este relato apresenta contribuições e implicações para a prática, o planejamento e o desenvolvimento de modificações nos serviços ambulatoriais que utilizam o MACC como modelo de atenção. Serviços esses que, mesmo em meio a pandemia, precisaram manter o acompanhamento e monitoramento da saúde das pessoas com condições crônicas complexas, minimizando as descompensações e, consequentemente, diminuindo a necessidade de essas pessoas utilizarem os serviços de saúde.

\section{CONTRIBUIÇÕES DOS AUTORES}

Desenho do relato de experiencia. Francielle Renata Danielli Martins Marques. Maria Aparecida Salci. Lígia Carreira.

Levantamento de informações. Francielle Renata Danielli Martins Marques.

Análise da experiencia. Francielle Renata Danielli Martins Marques. Lilian Ferreira Domingues.

Interpretação. Francielle Renata Danielli Martins Marques.

Lilian Ferreira Domingues. Maria Aparecida Salci. 
Redação e revisão crítica do manuscrito. Francielle Renata Danielli Martins Marques. Lilian Ferreira Domingues. Lígia Carreira. Maria Aparecida Salci.

Aprovação da versão final do artigo. Francielle Renata Danielli Martins Marques. Lilian Ferreira Domingues. Lígia Carreira. Maria Aparecida Salci.

Responsabilidade por todos os aspectos do conteúdo e a integridade do artigo publicado. Francielle Renata Danielli Martins Marques. Lilian Ferreira Domingues. Lígia Carreira. Maria Aparecida Salci.

\section{EDITOR ASSOCIADO}

Antonio José de Almeida Filho

\section{EDITOR CIENTÍFICO}

\author{
Ivone Evangelista Cabral 무
}

\section{REFERÊNCIAS}

1. World Health Organization. Origin of SARS-CoV-2 [Internet]. Geneva: WHO; 2020 [citado 2021 abr 20]. Disponível em: https://apps. who.int/iris/ bitstream/handle/10665/332197/WHO-2019-nCoV-FAQ-Virus_origin2020.1-eng.pdf

2. Rothan HA, Byrareddy SN. The epidemiology and pathogenesis of coronavirus disease (COVID-19) outbreak. J Autoimmun. 2020;109:102433. http://dx.doi.org/10.1016/j.jaut.2020.102433. PMid:32113704.

3. Ministério da Saúde (BR). Secretaria de Vigilância em Saúde. Boletim Epidemiológico Especial: Doença pelo Coronavírus COVID-10. Semana epidemiológica 25 [Internet]. Brasília: Ministério da Saúde; 2021 [citado 2021 jul 15]. Disponível em: https://www.gov.br/saude/pt-br/ media/pdf/2021/julho/02/69_boletim_epidemiologico_covid_2junho. pdf?dLDf=false

4. Dal Pai D, Gemelli MP, Boufleuer E, Finckler PVPR, Miorin JD, Tavares JP et al. Repercussões da pandemia pela COVID-19 no serviço pré-hospitalar de urgência e a saúde do trabalhador. Esc Anna Nery. 2021;25(spe):e20210014. http://dx.doi.org/10.1590/2177-9465ean-2021-0014.

5. Liu N, Huang R, Baldacchino T, Sud A, Sud K, Khadra M et al. Telehealth for noncritical patients with chronic diseases during the COVID-19 pandemic. J Med Internet Res. 2020;22(8):e19493. http://dx.doi. org/10.2196/19493. PMid:32721925.

6. Mendes EV. Desafios do SUS. Brasília, DF: CONASS; 2019. 869 p.

7. Santos CPRSD, Fernandes AFC, Silva DMD, Castro RCMB. Restructuring service at a mastology outpatient clinic during the COVID-19 pandemic. Rev Bras Enferm. 2021;74(74, Suppl 1):e20200571. http://dx.doi. org/10.1590/0034-7167-2020-0571. PMid:33533804.

8. Daltro MR, Faria AA. Relato de experiência: uma narrativa científica na pós-modernidade. Estud Pesqui Psicol. 2019;19(1):223-37. http:// dx.doi.org/10.12957/epp.2019.43015.

9. Marietto M. Observação participante e não participante: contextualização teórica e sugestão de roteiro para aplicação dos métodos. Rev Iberoam Estratég. 2018;17(4):5-18. http://dx.doi.org/10.5585/ijsm.v17i4.2717.

10. Consórcio Intermunicipal de Saúde do Vale do Ivaí e Região. História [Internet]. 2021 [citado 2021 nov 5]. Disponível em: http://www.cisvir. com.br/cisvir/index.php/historia/

11. Secretaria de Estado de Saúde do Paraná (PR). Nota Orientativa $\mathrm{n}^{\circ}$ 33/2020. Nota Orientativa para os ambulatórios organizados no Modelo de Atenção às Condições Crônicas em tempos de COVID 19 [Internet]. 2020 [citado 2021 abr 25]. Disponível em: https://coronavirus.ceara.gov. br/wp-content/uploads/2020/06/03.06_Nota-Tecnica-Ambulatorial_V1.pdf

12. Agência Nacional de Vigilância Sanitária. Nota técnica GVIMS/GGTES/ ANVISA nํ0 04/2020. Orientações para serviços de saúde: medidas de prevenção e controle que devem ser adotadas durante a Assistência aos casos suspeitos ou confirmados de infecção pelo novo coronavírus (SARS-CoV-2) [Internet]. 2020 [citado 2021 nov 4]. Disponível em: https:// portaldeboaspraticas.iff.fiocruz.br/biblioteca/nota-tecnica-no-04-2020/

13. Schiffer L, Gertges R, Nöhre M, Schieffer E, Tegtbur U, Pape L et al. Use and preferences regarding internet-based health care delivery in patients with chronic kidney disease. BMC Med Inform Decis Mak. 2021;21(1):34 http://dx.doi.org/10.1186/s12911-020-01375-9. PMid:33522934.

14. Secretaria de Estado de Saúde do Paraná (PR). Nota Orientativa no 04/2020. Atendimento dos idosos frente à pandemia COVID-19 [Internet]. 2020 [citado 2021 abr 28]. Disponível em: https://www.saude. pr.gov.br/sites/default/arquivos_restritos/files/documento/2020-06/ no_04_atendimento_dos_idosos_frente_a_pandemia_covid_19.pdf 